\title{
Avaliação da Alexitimia em Crianças Portuguesas com Perturbação de Hiperatividade/Défice de Atenção
}

\section{Assessment of Alexithymia in Portuguese Children with Attention Deficit Hyperactivity Disorder}

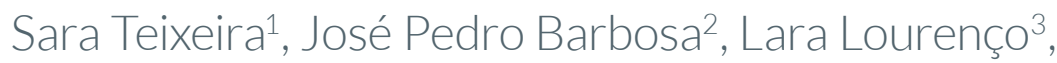
Daniel Gonçalves ${ }^{3}$, Micaela Guardiano ${ }^{3}$

\section{RESUMO}

INTRODUÇÃO: Diversos estudos têm demonstrado existir uma perturbação do processamento emocional em crianças com perturbação hiperatividade/ défice de atenção (PHDA). No entanto, existem poucos estudos relativos à associação entre alexitimia e PHDA tanto em adultos como em crianças, e nenhum na população portuguesa.

MÉTODOS: A amostra acidental inclui 50 crianças previamente diagnosticadas com PHDA e sob tratamento e 51 crianças saudáveis sem PHDA, com idades compreendidas entre os 8 e os 17 anos. Com o intuito de avaliar a alexitimia foi aplicada a versão portuguesa do questionário de alexitimia para crianças.

RESULTADOS: Comparando os dois grupos, não se verificaram diferenças significativas em nenhum dos três fatores da escala de alexitimia: dificuldade em identificar sentimentos, dificuldade em descrever sentimentos e pensamento orientado externamente, assim como na pontuação total da escala.

DISCUSSÃO: No nosso estudo, crianças com PHDA sob intervenção farmacológica e psicoterapêutica apresentaram níveis de alexitimia sobreponíveis à população controlo.

CONCLUSÃO: O nosso estudo pretende reforçar a importância da avaliação do processamento emocional na PHDA e a sua relação com as intervenções terapêuticas realizadas, abrindo portas a que outras investigações se dediquem a este tema.

PALAVRAS-CHAVE: Criança; Emoções; Perturbação de Hiperatividade com Défice de Atenção; Sintomas Afetivos

\section{ABSTRACT}

INTRODUCTION: Various studies have demonstrated the existence of impairment in emotional processing in children with attention deficit hyperactivity disorder (ADHD). However, there are few studies regarding the association between alexithymia and ADHD, both in adults and children, and none in the Portuguese population. 
METHODS: The accidental sample included 50 children who had been previously diagnosed with ADHD and were undergoing treatment, as well as 51 healthy children without ADHD, aged 8 to 17. To assess alexithymia, the Portuguese version of the Alexithymia Questionnaire for Children was used.

RESULTS: Comparing the two groups, there were no significant differences in any of the three factors-difficulty in identifying feelings, difficulty in describing feelings and externally oriented thinking, or in the total score.

DISCUSSION: In our study, children with ADHD who were undergoing pharmacological treatment and psychotherapy had alexithymia scores similar to those in the control group.

CONCLUSION: Our study intends to reinforce the importance of emotional processing evaluation in ADHD and its relationship with therapeutic interventions, opening the door for further insight into this issue.

KEYWORDS: Affective Symptoms; Attention Deficit Disorder with Hyperactivity; Child; Emotions

\section{INTRODUÇÃO}

A alexitimia resulta de um défice no processamento da reação emocional com dificuldades no reconhecimento e na expressão dos sentimentos experienciados. Traduz-se numa capacidade limitada para refletir e assim regular diretamente ou indiretamente as emoções. ${ }^{1}$ Como consequência deste défice observa-se também uma dificuldade em comunicar verbalmente as emoções aos outros. $^{2}$

Até aos dias de hoje poucos estudos analisaram a alexitimia na infância e na adolescência. Os estudos existentes utilizaram medidas semelhantes às do adulto e mostraram que a dificuldade em exprimir sentimentos está relacionada com estados emocionais e físicos como obesidade, stress pós-traumático, perturbações do comportamento alimentar, farmacodependência e perturbação bipolar, entre outros., ${ }^{3,4}$ Por outro lado, verificou-se que adolescentes com níveis mais baixos de ansiedade ou depressão e maior satisfação com a vida apresentavam igualmente valores mais baixos de alexitimia. ${ }^{5}$

A perturbação de hiperatividade/ défice de atenção (PHDA) é a perturbação neuro-comportamental mais frequente nas crianças, sendo a sua etiologia multifatorial. Pensa-se que resulta de uma interação complexa de fatores genéticos/ hereditários, neurobiológicos e ambientais. ${ }^{6}$ Relativamente aos fatores neurológicos, estabeleceu-se uma correlação entre PHDA e alterações nos lobos pré-frontais, mais especificamente no córtex pré-frontal.7 Estas mesmas regiões do cérebro estão associadas a alterações comportamentais relatadas por António Damásio e que estão intimamente associadas ao circuito das emoções. ${ }^{8}$ Estas alterações neurológicas podem ser corrigidas através da terapêutica farmacológica, o que sustenta a etiologia da perturbação, não se tratando apenas de um problema de educação ou cultural.7 A presença de alterações em várias regiões cerebrais implicadas na regulação emocional, em crianças com PHDA, sugere a inclusão de alterações emocionais como parte da sintomatologia de base da PHDA.,10 Alguns estudos defendem que as disfunções detetadas no reconhecimento emocional, nomeadamente de expressões faciais e da prosódia, não estão relacionadas com as deficiências cognitivas características da PHDA, mas constituem um défice primário. ${ }^{11,12}$

O estudo de Williams apresenta dados especialmente valiosos, já que é uma investigação pioneira na exploração dos mecanismos neurológicos implícitos no processamento emocional na PHDA. Os resultados deste estudo indicam a presença de importantes anomalias eletrofisiológicas nas provas de reconhecimento de emoções básicas em faces humanas (especialmente quando estas são negativas, tais como o medo e a raiva) em crianças e adolescentes com PHDA. Tais anomalias são particularmente marcadas em níveis precoces do processamento emocional e refletem-se numa redução significativa da componente occipital. Esse estudo sugere que alterações precoces em áreas visuais occipitais poderiam relacionar-se com um funcionamento anómalo da amígdala, já que esta é capaz de modular a atividade das áreas sensoriais através das suas projeções no córtex visual e auditivo. Para além disso o estudo vem confirmar o benefício da terapêutica com metilfenidato na normalização das referidas anomalias. ${ }^{13}$

A PHDA é uma perturbação heterogénea com diversas comorbilidades associadas, por este motivo é imperativo realizar uma avaliação minuciosa que integre variáveis neuropsicológicas. Caracteriza-se por falta de atenção e/ou impulsividade-hiperatividade, que deverá ser mais marcada do que a observada num indivíduo sem PHDA, com o mesmo nível de desenvolvimento. É uma das condições neurodesenvolvimentais mais comuns e investigadas da infância, cujos sintomas têm particular impacto nas crianças em idade escolar. ${ }^{14}$

São reconhecidas nestas crianças dificuldades a nível do autocontrolo, labilidade emocional e dificuldades nos relacionamentos sociais. ${ }^{15,16}$ 
Num estudo realizado em crianças italianas, utilizando a versão italiana do Questionário de Alexitimia para crianças, foram identificados níveis de alexitimia superiores em crianças com PHDA, antes do início da intervenção terapêutica. ${ }^{17}$ No nosso estudo optamos por realizar a mesma avaliação em crianças sob intervenção farmacológica e comportamental.

Apesar de vários estudos sugerirem que na PHDA existem défices em domínios relacionados com a emoção, nenhum estudo analisou a associação entre a alexitimia e a PHDA em crianças portuguesas. A investigação em Portugal parece ter-se concentrado nos aspetos cognitivos da PHDA ${ }^{18,19}$ e menos nas alterações emocionais. ${ }^{20}$

Desta forma, o presente estudo tem como finalidade:

- Investigar a relação entre alexitimia e perturbação de hiperatividade-défice de atenção numa amostra de crianças portuguesas com idades compreendidas entre os 8 e os 17 anos, sob terapêutica farmacológica e comportamental.

- Sublinhar a importância das alterações emocionais na PHDA e a importância da intervenção terapêutica na sua regulação.

- Incentivar ao desenvolvimento de novas investigações que abordem o estudo das emoções na PHDA.

Neste estudo, testamos a hipótese de que crianças com PHDA com boa resposta ao tratamento farmacológico e comportamental apresentem não só melhoria ao nível dos sintomas centrais da PHDA, mas também melhoria da regulação emocional.

\section{MATERIAL E MÉTODOS}

\section{AMOSTRA}

A amostra acidental inclui 101 crianças (58 do sexo masculino e 43 do sexo feminino) com idades compreendidas entre os 8 e os 17 anos. Destas 101 crianças, 50 são acompanhadas na consulta de Pediatria do Neurodesenvolvimento do Centro Hospitalar Universitário de São João - Porto, previamente diagnosticadas com perturbação de hiperatividade/défice de atenção estando já sob acompanhamento médico e psicológico. $\bigcirc$ grupo controlo é constituído por crianças sem o diagnóstico de PHDA, das quais 24 crianças pertenciam a um agrupamento de escuteiros, 27 crianças pertenciam a um Centro de Estudos do distrito do Porto. Deste grupo foram excluídas crianças com diagnóstico de PHDA, com alterações do comportamento e com perturbação do desenvolvimento intelectual. Em nenhum dos participantes estava diagnosticada qualquer outra patologia.

A investigação foi aprovada pela Comissão de Ética do Centro Hospitalar do São João. A participação das crianças foi autorizada pelos seus representantes legais, mediante assinatura do consentimento informado e nenhuma criança se recusou a responder ao questionário.

\section{MATERIAL}

\section{Diagnóstico de PHDA}

Para o diagnóstico de PHDA foram utilizados os critérios de diagnóstico incluídos no Manual de Diagnóstico e Estatística das Perturbações Mentais (DSM-5, 2014), ${ }^{21}$ tendo por base a avaliação da criança, história clínica e questionários de Conners, versões para pais e professores. ${ }^{22}$

\section{Avaliação da Alexitimia}

Com o intuito de avaliar a alexitimia foram desenvolvidos diversos instrumentos, nomeadamente a Escala de Alexitimia de Toronto (TAS-20), sendo esta a mais reconhecida e usada tanto a nível de investigação como na prática clínica. ${ }^{23}$ A TAS-20 foi desenvolvida em 1992 por Bagby, Taylor e Parker, sendo as suas propriedades psicométricas estudadas continuamente. A estrutura desta escala foi criada de modo a refletir o constructo de alexitimia tal como foi inicialmente proposto por Nemiah em $1970 .{ }^{24}$

A TAS-20 é um instrumento fiável e bem validado ${ }^{25}$ que faculta uma pontuação global em alexitimia, bem como pontuações referidas aos três fatores que extraem os componentes mais representativos do constructo, nomeadamente, dificuldade em identificar sentimentos (DIF), dificuldade em descrever sentimentos (DDS) e pensamento orientado externamente (POE).

Trata-se de um questionário de autoavaliação com 20 itens, onde a pessoa regista o seu grau de concordância para cada um dos itens numa escala de Likert de cinco pontos, variando entre 1 - Discordo Totalmente e 5 Concordo Totalmente. Esta escala foi traduzida por diversos países, incluindo Portugal, onde foi validada no ano de 2000 por Praceres, Parker e Taylor.2,26

○ Questionário de Alexitimia para Crianças (QAC) 27 é uma adaptação do TAS-20. Neste foram mantidos os 20 itens do TAS-20 adaptados à linguagem da população infantil, como por exemplo "Mais do que limitar-me a descrever os problemas, prefiro analisá-los" passou a "Quando tenho um problema, quero saber de onde o problema vem, e não apenas falar sobre ele", "É essencial manter contacto com as emoções" passou a "É importante perceber como me sinto por dentro" e por fim "Prefiro ver programas leves que distraiam do que dramas psicológicos" passou a "Na TV prefiro ver programas de diversão que me distraiam do que histórias sobre os problemas dos outros". 
Para a dificuldade em identificar sentimentos (DIF) contribuem as questões 1, 3, 6, 7, 9, 13 e 14. As questões 2, 4, 11, 12 e a 17 contribuem para a dificuldade em descrever sentimentos (DDS). Por último, as questões 5 , 8, 10, 15, 16, 18, 19 e 20 contribuem para a escala de pensamento orientado externamente (POE). Contudo, para efeitos somatórios das pontuações individuais as questões 4, 5, 10, 18 e 19 são pontuadas inversamente.

A escala de Likert de cinco pontos foi convertida numa escala de três pontos, onde o 0 corresponde a "não é verdade", 1 a "pouco verdade" e 2 a "verdade". ${ }^{27}$

No instrumento, não há um cut-off definido para considerar o indivíduo alexitímico. Esta definição surge em comparação com o grupo controlo.

Rieffe, Oosterveld e Terwogt ${ }^{27}$ validaram a QAC e identificaram a presença de alexitimia também em crianças. Estes investigadores destacaram a importância dos fatores "Dificuldade em Identificar Sentimentos" e "Dificuldade em Descrever Sentimentos" na identificação da alexitimia em crianças. Quanto ao "Pensamento orientado para o exterior" os autores mostraram alguma relutância em relação aos seus resultados dada a sua fraca consistência.

Para esta investigação foi elaborada uma versão adaptada da versão portuguesa da escala de alexitimia de Toronto de 20 itens $^{2}$ e do "Alexithymia questionnaire for children" 27 culminando no questionário que se apresenta em anexo.

\section{ANÁLISE ESTATÍSTICA}

Utilizou-se o software Statistical Package for the Social Sciences (SPSS), versão 25.0, para a realização da análise estatística.

Para a verificação da existência de diferenças significativas entre o grupo de crianças com PHDA e o grupo de controlo, utilizou-se o teste $U$ de Mann-Whitney, no caso das variáveis contínuas, e o teste do $x^{2}$ - qui-quadrado, no caso das variáveis categóricas, recorrendo-se ao teste exato de Fisher em caso de falha no cumprimento dos pressupostos de contagens esperadas mínimas. Para efeitos de significância estatística, foram conside-

TABELA 1. Consistência interna do Questionário de Alexitimia para crianças.

\begin{tabular}{l|l}
\hline SUBESCALA & $a$ DE CRONBACH \\
\hline DIF & 0,794 \\
\hline DDS & 0,593 \\
\hline POE & 0,351 \\
\hline TOTAL & 0,750 \\
\hline
\end{tabular}

rados significativos os valores de $p$ iguais ou inferiores a 0,05 . A consistência interna do questionário foi avaliada usando o teste $p$ de Cronbach. Ainda para avaliar a coesão da escala, foi investigada a correlação p de Spearman entre os resultados de cada subescala e a pontuação final do questionário.

\section{RESULTADOS}

A nossa amostra incluiu 101 crianças, das quais 50 tinham um diagnóstico de PHDA e 51 faziam parte de um grupo controlo.

O questionário apresentou uma consistência interna adequada quando avaliado no seu todo - a 0,750 e nas subescalas Dificuldade em Identificar Sentimentos (DIF) e Dificuldade em Descrever Sentimentos (DDS). Pelo contrário, a consistência interna da subescala Pensamento Orientado Externamente (POE) mostra-se medíocre, como se vê na Tabela 1.

Na Tabela 2 apresentam-se os valores da correlação entre os valores de cada dimensão do QAC e a pontuação total, assim como das três dimensões entre si.

Considerando a amostra no seu todo, obtivemos uma média de idades de 13,09 anos ( $D P=2,478)$, uma escolaridade média de 7,68 anos ( $\mathrm{DP}=2,37)$, uma distribuição por sexos de 58/43 (Masculino/Feminino), e as seguintes pontuações no questionário: total-média de 16,85 $(\mathrm{DP}=6,02)$; dificuldade em identificar sentimentos (DIF)-média de 5,37 ( $D P=3,32)$; dificuldade em descrever sentimentos (DDS)-média de 4,43 (DP=2,33); pensamento orientado externamente (POE) - média de $7,05(\mathrm{DP}=2,42)$.

Comparando os dois grupos, em termos demográficos, não se verificam diferenças significativas na idade ou escolaridade, mas o mesmo não ocorre na distribuição por sexo (Tabela 3). Quanto às pontuações do questionário, não se verificam diferenças significativas em nenhum dos três fatores, assim como na pontuação total (Tabela 5). Não há diferenças significativas na escolaridade da

TABELA 2. Correlações de Spearman entre as subescalas e a pontuação total do Questionário de Alexitimia para crianças.

\begin{tabular}{|l|l|}
\hline SUBESCALA & CORRELAÇÃO DE SPEARMAN $(p)$ \\
\hline DIF/TOTAL & $0,859^{*}$ \\
\hline DDS/TOTAL & $0,788^{*}$ \\
\hline POE/TOTAL & $0,538^{*}$ \\
\hline DIF/DDS & $0,638^{*}$ \\
DDS/POE & 0,166 \\
\hline DIF/POE & 0,172 \\
\hline
\end{tabular}

*correlação significativa para um nível de significância de 0,05 
TABELA 3. Caracterização demográfica da amostra.

\begin{tabular}{|c|c|c|c|c|}
\hline & $\begin{array}{l}\text { CRIANÇAS COM PHDA } \\
(n=50)\end{array}$ & $\begin{array}{l}\text { GRUPO DE CONTROLO } \\
(n=51)\end{array}$ & TOTAL $(n=101)$ & $p$ \\
\hline \multicolumn{5}{|l|}{ SEXO } \\
\hline MASCULINO & 39 (78\%) & $19(37,3 \%)$ & $58(57,4 \%)$ & \multirow{2}{*}{$\begin{array}{c}<0,001 \\
\left(T E S T E X^{2}\right)\end{array}$} \\
\hline FEMININO & $11(22 \%)$ & $32(62,7 \%)$ & $43(42,6 \%)$ & \\
\hline \multicolumn{5}{|l|}{ GRUPO ETÁRIO } \\
\hline $8-10$ & $6(12 \%)$ & $13(25,5 \%)$ & $19(18,9 \%)$ & \multirow{3}{*}{$\begin{array}{c}0,131 \\
\left(\text { TESTE } X^{2}\right)\end{array}$} \\
\hline $11-14$ & $27(54 \%)$ & $19(37,2 \%)$ & $2(45,6 \%)$ & \\
\hline $15-17$ & $17(34 \%)$ & $19(37,2 \%)$ & $12(35,6 \%)$ & \\
\hline \multicolumn{5}{|l|}{ ESCOLARIDADE } \\
\hline $1^{\circ} \mathrm{CICLO}$ DO ENSINO BÁSICO & $3(6 \%)$ & $6(11,8 \%)$ & $9(8,9 \%)$ & \multirow{4}{*}{$\begin{array}{c}\text { 0,144 } \\
\text { (TESTE } \\
\text { EXATO DE } \\
\text { FISHER) }\end{array}$} \\
\hline $2^{\circ} \mathrm{CICLO}$ DO ENSINO BÁSICO & $12(24 \%)$ & $11(21,5 \%)$ & $23(22,8 \%)$ & \\
\hline $3^{\circ} \mathrm{CICLO}$ DO ENSINO BÁSICO & $27(38 \%)$ & $18(35,3 \%)$ & $45(44,6 \%)$ & \\
\hline ENSINO SECUNDÁRIO & $8(16 \%)$ & $16(31,3 \%)$ & $24(23,8 \%)$ & \\
\hline
\end{tabular}

TABELA 4. Escolaridade dos pais de acordo com os grupos.

\begin{tabular}{|c|c|c|c|c|}
\hline & $\begin{array}{l}\text { CRIANÇAS COM } \\
\text { PHDA }(n=50)\end{array}$ & $\begin{array}{l}\text { GRUPO DE CON- } \\
\text { TROLO }(n=51)\end{array}$ & TOTAL $(n=101)$ & $p$ \\
\hline \multicolumn{5}{|l|}{ ESCOLARIDADE DO PAI } \\
\hline 1. ${ }^{\circ}$ CICLO DO ENSINO BÁSICO & $8(16 \%)$ & $4(7,8 \%)$ & $12(11,9 \%)$ & \multirow{6}{*}{$\begin{array}{c}0,051 \\
\left(\text { TESTE } X^{2}\right)\end{array}$} \\
\hline 2. ${ }^{\circ} \mathrm{CICLO}$ DO ENSINO BÁSICO & $17(34 \%)$ & $9(17,6 \%)$ & $26(25,7 \%)$ & \\
\hline 3. ${ }^{\circ}$ CICLO DO ENSINO BÁSICO & $15(30 \%)$ & $15(29,4 \%)$ & $30(29,7 \%)$ & \\
\hline ENSINO SECUNDÁRIO & $6(12 \%)$ & $17(33,3 \%)$ & $23(22,8 \%)$ & \\
\hline BACHARELATO & $\mathrm{O}(0 \%)$ & $\mathrm{O}(0 \%)$ & $\mathrm{O}(0 \%)$ & \\
\hline LICENCIATURA & $4(8 \%)$ & $6(11,8 \%)$ & $10(9,9 \%)$ & \\
\hline \multicolumn{5}{|l|}{ ESCOLARIDADE DA MÃE } \\
\hline 1. ${ }^{\circ}$ CICLO DO ENSINO BÁSICO & $9(18 \%)$ & $5(9,8 \%)$ & $14(13,9 \%)$ & \multirow{6}{*}{$\begin{array}{c}0,384 \\
\text { (TESTE EXATO DE } \\
\text { FISHER) }\end{array}$} \\
\hline 2. ${ }^{\circ}$ CICLO DO ENSINO BÁSICO & $8(16 \%)$ & $8(15,7 \%)$ & $16(15,8 \%)$ & \\
\hline 3. ${ }^{\circ} \mathrm{CICLO}$ DO ENSINO BÁSICO & $17(34 \%)$ & $13(25,5 \%)$ & $30(29,7 \%)$ & \\
\hline ENSINO SECUNDÁRIO & $12(24 \%)$ & $21(41,2 \%)$ & $33(32,7 \%)$ & \\
\hline BACHARELATO & $\mathrm{O}(0 \%)$ & $1(2 \%)$ & $1(1 \%)$ & \\
\hline LICENCIATURA & $4(8 \%)$ & $3(5,9 \%)$ & $7(6,9 \%)$ & \\
\hline
\end{tabular}

TABELA 5. Comparação de pontuações do Questionário de Alexitimia entre crianças com PHDA e sem PHDA, incluindo análise estratificada por sexos (teste U de Mann-Whitney).

\begin{tabular}{|c|c|c|c|}
\hline \multicolumn{4}{|c|}{ SEXO FEMININO } \\
\hline SUBESCALAS & CRIANÇAS COM PHDA (n=11) & GRUPO DE CONTROLO (n=32) & $p$ \\
\hline DIF & $5,36 \pm 2,94$ & $6,56 \pm 3,09$ & 0,313 \\
\hline DDS & $4,54 \pm 2,07$ & $4,81 \pm 2,47$ & 0,811 \\
\hline POE & $7,09 \pm 3,02$ & $7,13 \pm 2,28$ & 0,911 \\
\hline PONTUAÇÃO & $17 \pm 6,80$ & $18,5 \pm 5,59$ & 0,558 \\
\hline \multicolumn{4}{|c|}{ SEXO MASCULINO } \\
\hline SUBESCALAS & CRIANÇAS COM PHDA (n=39) & GRUPO DE CONTROLO (n=19) & $p$ \\
\hline DIF & $4,79 \pm 3,44$ & $4,53 \pm 3,29$ & 0,764 \\
\hline DDS & $4,21 \pm 2,40$ & $4,21 \pm 2,15$ & 0,980 \\
\hline POE & $7,08 \pm 2,50$ & $6,84 \pm 2,29$ & 0,940 \\
\hline PONTUAÇÃO & $16,08 \pm 6,14$ & $15,58 \pm 5,87$ & 0,778 \\
\hline \multicolumn{4}{|l|}{ TOTAL } \\
\hline SUBESCALAS & CRIANÇAS COM PHDA $(n=50)$ & GRUPO DE CONTROLO $(n=51)$ & $p$ \\
\hline DIF & $4,92 \pm 3,32$ & $5,80 \pm 3,29$ & 0,214 \\
\hline DDS & $4,28 \pm 2,31$ & $4,59 \pm 2,35$ & 0,609 \\
\hline POE & $7,08 \pm 2,59$ & $7,02 \pm 2,27$ & 0,918 \\
\hline PONTUAÇÃO & $16,28 \pm 6,23$ & $17,41 \pm 5,81$ & 0,410 \\
\hline
\end{tabular}


mãe entre os dois grupos. A diferença na escolaridade do pai apresenta um valor p de 0,051 (Tabela 4).

Não houve diferenças significativas entre os dois grupos, quer em qualquer um dos três fatores, quer na pontuação total. Isto verificou-se tanto na amostra completa como na análise estratificada por sexos (Tabela 5).

\section{DISCUSSÃO}

Na nossa amostra, comparando os dois grupos, em função das variáveis demográficas, não se verificam diferenças significativas. Verifica-se, no entanto, que a composição por sexos nos dois grupos é significativamente diferente nos dois grupos, sendo mais representado o sexo masculino no grupo com PHDA do que no grupo de controlo. Este achado está de acordo com a bibliografia, uma vez que a PHDA é três a quatro vezes mais comum no sexo masculino (ratio variável de 2,5-9:1, consoante a população em estudo). ${ }^{28}$

Entre outros, são considerados fatores de risco familiares para a PHDA, o divórcio ou conflito parental, depressão materna e baixo nível socioeconómico e cultural. ${ }^{29}$ Pela análise dos dados, verificou-se que, nesta amostra, os pais das crianças diagnosticadas com PHDA tinham níveis de escolaridade mais baixo, em comparação com a escolaridade dos pais do grupo controlo, apesar de não haver diferença estatisticamente significativa que permita uma generalização (Tabela 4).

Com o objetivo de avaliar a alexitimia em crianças com PHDA e num grupo controlo utilizamos o Questionário de Alexitimia para Crianças. Este demonstrou uma consistência interna adequada quando avaliado no seu todo e nas subescalas de DIF e DDS, mas não na subescala POE. Os resultados do presente estudo estão de acordo com a revisão da literatura que relata fraca consistência da subescala POE principalmente nos grupos mais jovens. ${ }^{30}$ No que diz respeito aos resultados das subescalas e total do QAC, não se verificam diferenças estatisticamente significativas entre o grupo controlo e o grupo com PHDA.

É reconhecido que a labilidade emocional em crianças com PHDA é 10 vezes maior (38\%) ${ }^{31}$ do que a população geral contribuindo para a disfunção atribuída a esta complexa perturbação também é, cada vez mais, compreendido o impacto da terapêutica farmacológica, não apenas nos sintomas centrais, mas também na regulação emocional. De facto, o metilfenidato (MPH) ao normalizar a ativação dos circuitos fronto-estriatais, incluindo o córtex frontal inferior direito, o córtex cingulado e o núcleo estriado, ${ }^{32,33}$ otimizando os níveis de catecolaminas nessas regiões têm efeitos positivos no controlo da aten- ção e na impulsividade, mas também na regulação de emoções negativas. ${ }^{34,35}$ Tal é reforçado por um estudo realizado em jovens universitários com PHDA que revelou que a terapêutica com psicoestimulantes melhorou significativamente os sintomas centrais da perturbação, mas também a alexitimia. ${ }^{36} \bigcirc$ papel positivo do tratamento com psicoestimulantes é também reconhecido na prevenção das perturbações emocionais. ${ }^{37}$

A atomoxetina mostrou também ser útil para reduzir a labilidade emocional em adultos com PHDA. ${ }^{38}$

Consideramos, pois, que o nosso estudo reforça os resultados de outros trabalhos anteriores, alertando para a importância do tratamento da PHDA, não apenas ao nível da melhoria do funcionamento executivo, mas também na regulação emocional.

No entanto, este trabalho apresenta diversas limitações. Uma destas limitações prende-se com o tipo e a dimensão da amostra que pode limitar a robustez dos valores encontrados.

Por outro lado, apesar de num cômputo global o QAC ser bastante fiável, a deficiente consistência interna da subescala Pensamento orientado externamente - (POE) pode ter prejudicado os nossos resultados.

Outras das limitações deste estudo é ser um estudo transversal, isto é, todas as medições foram feitas num único momento não existindo, portanto, períodos diferentes de avaliação dos indivíduos, nomeadamente antes e após o início da intervenção terapêutica.

De facto, por se tratar de uma avaliação transversal, optamos por avaliar a alexitimia em crianças sob intervenção farmacológica e comportamental e com estabilidade clínica.

Por fim, numa futura investigação observar o grupo em dois períodos, antes e depois da intervenção terapêutica, poderá trazer resultados adicionais.

Um estudo recente aponta as dificuldades na regulação emocional e autoconceito como défices centrais na PHDA do adulto. ${ }^{39}$ Será fundamental que, também em idade pediátrica, futuras investigações se dediquem à avaliação das competências emocionais no âmbito desta desafiante perturbação. ${ }^{40}$

\section{CONCLUSÃO}

Comparativamente a outros estudos em crianças e adultos com PHDA sem intervenção farmacológica, na nossa amostra não se encontraram níveis de alexitimia superiores ao grupo controlo. O nosso estudo pretende reforçar a importância da avaliação do processamento 
emocional na PHDA e a sua relação com as intervenções terapêuticas realizadas, abrindo portas a que outras investigações se dediquem a este tema.

\section{AGRADECIMENTOS}

Agradeço ao Professor Victor Viana pela visão crítica e oportuna, pelo empenho inexcedível e saudavelmente exigente, o que contribuiu para enriquecer o trabalho realizado.

CONFLITOS DE INTERESSE: Os autores declaram a inexistência de conflitos de interesse na realização do presente trabalho.

FONTES DE FINANCIAMENTO: Não existiram fontes externas de financiamento para a realização deste artigo.

CONFIDENCIALIDADE DOS DADOS: Os autores declaram ter seguido os protocolos da sua instituição acerca da publicação dos dados de doentes.

PROTEÇÃO DE PESSOAS E ANIMAIS: Os autores declaram que os procedimentos seguidos estavam de acordo com os regulamentos estabelecidos pelos responsáveis da Comissão de Investigação Clínica e Ética e de acordo com a Declaração de Helsínquia da Associação Médica Mundial.

PROVENIÊNCIA E REVISÃO POR PARES: Não comissionado; revisão externa por pares.

CONFLICTS OF INTEREST: The authors have no conflicts of interest to declare.

FINANCING SUPPORT: This work has not received any contribution, grant or scholarship.

CONFIDENTIALITY OF DATA: The authors declare that they have followed the protocols of their work center on the publication of data from patients.

PROTECTION OF HUMAN AND ANIMAL SUBJECTS: The authors declare that the procedures followed were in accordance with the regulations of the relevant clinical research ethics committee and with those of the Code of Ethics of the World Medical Association (Declaration of Helsinki).

PROVENANCE AND PEER REVIEW: Not commissioned; externally peer reviewed.

\section{REFERÊNCIAS}

1. Lewis M, Haviland-Jones JM, Barret LF, editors. Handbook of Emotions. 4th ed. New York: Guilford Press; 2018.

2. Verissimo R. Versão portuguesa da escala de alexitimia de toronto de 20- itens. Adaptação linguística, validação semântica e estuda de fiabilidade. Acta Med Port. 2001;14:529-36.

3. Waller E, Scheidt CE. Somatoform disorders as disorders of affect regulation: A development perspective. Int Rev Psychiatry. 2006;18:13-24.
4. Masi G, Muratori P, Manfredi A, Pisano S, Milone A. Child behaviour checklist emotional dysregulation profiles in youth with disruptive behaviour disorders: Clinical correlates and treatment implications. Psychiatry Res. 2015;225:191-9. doi: 10.1016/j.psychres.2014.11.019.

5. Extremera N, Durán A, Rey L. Perceived emotional intelligence and dispositional optimism-pessimism: Analyzing their role in predicting psychological adjustment among adolescents. Pers Individ Dif. 2007;42:1069-79.

6. Barkley RA. Attention-Deficit Hyperactivity Disorder: A Handbook for Diagnosis and Treatment. 4th ed. New York: Guilford Press; 2015.

7. Barkley RA. Attention-Deficit Hyperactivity Disorder: A Handbook for Diagnosis and Treatment. 2nd ed. New York: Guilford Press; 1998.

8. Damásio A. O Sentimento de Si: O Corpo, emoção e a neurobiologia da consciência. Lisboa: Publicações Europa-América; 2000.

9. Martel MM. Research Review: A new perspective on attention-deficit/hyperactivity disorder: emotion dysregulation and trait models. J Child Psychol Psychiatry. 2009:50:1042-51. doi: 10.1111/j.1469-7610.2009.02105.x.

10. Villemonteix T, Purper-Ouakil D, Romo L. La dysrégulation émotionnelle est-elle une des composantes du trouble déficit d'attention/hyperactivité? Encephale. 2015;41:108-14.

11. Rodrigo-Ruiz D, Pérez-González JC, Cejudo J. Dificultades de reconocimiento emocional facial como déficit primario en niños con trastorno por déficit de atención/hiperactividad: Revisión sistemática. Rev Neurol. 2017;65:145-52.

12. Albert J, López-Martín S, Fernández-Jaén A, Carretié L. Alteraciones emocionales en el trastorno por déficit de atención/hiperactividad: datos existentes y cuestiones abiertas TT - Emotional alterations in attention deficit hyperactivity disorder: Existing data and open questions. Rev Neurol. 2008;47:39-45.

13. Williams LM, Hermens DF, Palmer D, Kohn M, Clarke S, Keage $\mathrm{H}$, et al. Misinterpreting emotional expressions in attention-deficit/hyperactivity disorder: evidence for a neural marker and stimulant effects. Biol Psychiatry. 2008;63:917-26. doi: 10.1016/j.biopsych.2007.11.022.

14. Daley D, Jones K, Hutchings J, Thompson M. Attention deficit hyperactivity disorder in pre-school children: current findings, recommended interventions and future directions. Child Care Health Dev. 2009;35:754-66. doi: 10.1111/j. 1365-2214.2009.00938.x.

15. Trott G-E. Attention-deficit/hyperactivity disorder (ADHD) in the course of life. Eur Arch Psychiatry Clin Neurosci. 2006;256(S1):i21-i25.

16. Classi P, Milton D, Ward S, Sarsour K, Johnston J. Social and emotional difficulties in children with ADHD and the impact on school attendance and healthcare utilization. Child Adolesc Psychiatry Ment Health. 2012;6:33.

17. Donfrancesco R, Di Trani M, Gregori P, Auguanno G, Melegari MG, Zaninotto S, et al. Attention-deficit/hyperactivity disorder and alexithymia: A pilot study. Atten Defic Hyperact Disord. 2013;5:361-7. doi: 10.1007/s12402-013-0115-9.

18. Guardiano M, Candeias L, Guimarães JE, Viana V, Almeida P. Avaliação da Leitura e Escrita de Crianças com Perturbação de Hiperactividade com Défice de Atenção. Acta Pediátrica Port. 2014:45:90-8.

19. Guardiano M, Candeias L, Eça Guimarães J, Viana V. Almeida P. Avaliação do processamento fonológico e da compreensão em crianças com PHDA. Psic Saúde Doenças. 2013;14:420-36.

20. Maia C, Guardiano M, Viana V, Almeida JP, Guimarães MJ. Auto-conceito em crianças com Hiperactividade e Défice de Atenção. Acta Med Port. 2011;24(S2):493-502. 
21. American Psychiatric Association. DSM-5 - Manual Diagnóstico e Estatística das Perturbações Mentais. 5a ed. Lisboa: Climepsi Editores; 2014.

22. Rodrigues A. Escalas de Conners para pais e professores: Tradução e adaptação para investigação. Lisboa: Departamento de Educação Especial e Reabilitação da Faculdade Motricidade Humana; 2000.

23. Freire L. Alexitimia: dificuldade de expressão ou ausência de sentimento? Uma análise teórica. Psicol Teor Pesqui. 2010; 26:15-24.

24. Nemiah JC. Affect and fantasy in patients with psychosomatic disorders. Mod Trends Psychosom Med. 1970;2:26-34.

25. Bagby RM, Parker JDA, Taylor GJ. The twenty-item Toronto Alexithymia scale-I. Item selection and cross-validation of the factor structure. J Psychosom Res. 1994;38:23-32.

26. Praceres N, Parker JDA, Taylor GJ. Adaptaçao Portuguesa da Escala de Alexitimia de Toronto de 20 Itens (tas-20) - Portuguese adaptation of de 20-item Toronto Alexithymia Scale ( tas-20 ). RIDEP. 2000;9:9-21.

27. Rieffe C, Oosterveld P, Terwogt MM. An alexithymia questionnaire for children: Factorial and concurrent validation results. Pers Individ Dif. 2006;40:123-133.

28. Rappley MD. Attention deficit-hyperactivity disorder. N Engl J Med. 2005;352:165-73.

29. Gatta M, Svanellini L, Sisti M, Battistella P, Simonelli A, Sudati $L$, et al. Emotional difficulties in adolescence: psychopathology and family interactions. Int Neuropsychiatr Dis J. 2015;4:47-54.

30. Loas G, Phanie Braun S, Delhaye M, Linkowski P. The measurement of alexithymia in children and adolescents: Psychometric properties of the Alexithymia Questionnaire for Children and the twenty-item Toronto Alexithymia Scale in different non-clinical and clinical samples of children and adolescents. PLoS One. 2017;12:1-18. doi: 10.1371/journal.pone.0177982.
31. Stringaris A, Goodman R. Mood lability and psychopathology in youth. Psychol Med. 2009;39:1237.

32. Wilens TE. Effects of methylphenidate on the catecholaminergic system in attention-deficit/hyperactivity disorder. J Clin Psychopharmacol. 2008;28:546-53.

33. Faraone SV. The pharmacology of amphetamine and methylphenidate: Relevance to the neurobiology of attention-deficit/ hyperactivity disorder and other psychiatric comorbidities. Neurosci Biobehav Rev. 2018;87:255-70. doi: 10.1016/j.neubiorev.2018.02.001.

34. Arnsten AFT, Pliszka SR. Catecholamine influences on prefrontal cortical function: Relevance to treatment of attention deficit/ hyperactivity disorder and related disorders. Pharmacol Biochem Behav. 2011;99:211-6. doi: 10.1016/j.pbb.2011.01.020.

35. Suzer Gamli I, Tahiroglu A. Six months methylphenidate treatment improves emotion dysregulation in adolescents with attention deficit/hyperactivity disorder: a prospective study. Neuropsychiatr Dis Treat. 2018;14:1329-37. doi: 10.2147/NDT.S164807.

36. Matuszak J, Miller G, Kemmelmeier M, Mason N. A pilot study of the impact of stimulant pharmacotherapy in college students with ADHD on alexithymia and psychological mindedness. Open J Med Psychol. 2013;2:139-142.

37. Biederman J, Monuteaux MC, Spencer T, Wilens TE, Faraone S V. Do stimulants protect against psychiatric disorders in youth with ADHD? A 10-year follow-up study. Pediatrics. 2009;124:71-8. doi: 10.1542/peds.2008-3347.

38. Moukhtarian TR, Cooper RE, Vassos E, Moran P, Asherson P. Effects of stimulants and atomoxetine on emotional lability in adults: A systematic review and meta-analysis. Eur Psychiatry. 2017;44:198-207. doi: 10.1016/j.eurpsy.2017.05.021.

39. Hirsch O, Chavanon M, Riechmann E, Christiansen H. Emotional dysregulation is a primary symptom in adult attention-deficit/ hyperactivity disorder (ADHD). J Affect Disord. 2018;232:41-7. doi: 10.1016/j.jad.2018.02.007.

40. Durlak JA, Domitrovich CE, Weissberg RP, Gullotta TP, editors. Handbook of Social and Emotional Learning: Research and practice. New York: The Guilford Press; 2015. 


\section{ANEXO 1 \\ QUESTIONÁRIO ALEXITIMIA}

Idade

Sexo: $M \square$ F

Ano de Escolaridade:

Escolaridade do Pai

Escolaridade da Mãe

Na próxima página vais encontrar 20 frases.

Cada uma delas diz respeito à maneira como as pessoas pensam relativamente aos seus próprios sentimentos. Deves assinalar em cada uma se o que lá diz acontece. FREQUENTEMENTE contigo, se apenas ÀS VEZES é verdade contigo, ou se NUNCA acontece contigo.

Escolhe a hipótese que mais se aproxima do que sentes. Só podes escolher uma hipótese.

Não existe nenhuma resposta certa ou errada, isto é apenas sobre a maneira como tu pensas.

\begin{tabular}{|c|c|c|c|c|}
\hline & & $\begin{array}{l}\text { NÃO É } \\
\text { VERDADE }\end{array}$ & $\begin{array}{c}\text { ÀS } \\
\text { VEZES }\end{array}$ & $\begin{array}{c}\text { SIM, } \\
\text { MUITAS } \\
\text { VEZES }\end{array}$ \\
\hline 1 & Muitas vezes sinto-me confuso em relação ao modo como me estou a sentir & & & \\
\hline 2 & É difícil encontrar as palavras certas para descrever como me sinto por dentro & & & \\
\hline 3 & Sinto algumas coisas no meu corpo que nem os médicos entendem & & & \\
\hline 4 & Sou capaz de descrever facilmente os meus sentimentos & & & \\
\hline 5 & $\begin{array}{l}\text { Quando tenho um problema, quero saber de onde o problema vem, } \\
\text { e não apenas falar sobre ele }\end{array}$ & & & \\
\hline 6 & Quando estou nervoso, não sei se me sinto triste, se assustado ou zangado & & & \\
\hline 7 & Fico muitas vezes baralhado com sensações que tenho no corpo & & & \\
\hline 8 & $\begin{array}{l}\text { Prefiro muito simplesmente deixar que as coisas aconteçam em vez de estar a } \\
\text { compreender porque é que se passaram assim }\end{array}$ & & & \\
\hline 9 & Por vezes não encontro palavras para explicar o que estou a sentir & & & \\
\hline 10 & É importante perceber como me sinto por dentro & & & \\
\hline 11 & Acho difícil descrever o que sinto em relação às outras pessoas & & & \\
\hline 12 & Dizem-me que deveria falar mais sobre o que estou a sentir & & & \\
\hline 13 & Não sei o que se passa cá dentro de mim & & & \\
\hline 14 & Muitas vezes não sei porque estou zangado & & & \\
\hline 15 & $\begin{array}{l}\text { Prefiro mais falar com as pessoas sobre coisas do seu dia-a-dia } \\
\text { do que sobre os seus sentimentos }\end{array}$ & & & \\
\hline 16 & $\begin{array}{l}\text { Na TV prefiro ver programas de diversão que me distraiam do que histórias } \\
\text { sobre os problemas dos outros }\end{array}$ & & & \\
\hline 17 & É-me difícil falar dos meus sentimentos, mesmo ao meu melhor amigo & & & \\
\hline 18 & $\begin{array}{l}\text { Posso sentir-me próximo de uma pessoa, mesmo quando estamos sentados, } \\
\text { sem falar, em silêncio }\end{array}$ & & & \\
\hline 19 & $\begin{array}{l}\text { Acho que pensar sobre os meus sentimentos é útil para resolver problemas } \\
\text { pessoais }\end{array}$ & & & \\
\hline 20 & $\begin{array}{l}\text { Quando tenho de me concentrar num filme para perceber a história, } \\
\text { vou gostar menos desse filme }\end{array}$ & & & \\
\hline
\end{tabular}

